\title{
Pulmonary and aortic root translocation in the management of transposition of the great arteries with ventricular septal defect and left ventricular outflow tract obstruction
}

Sheng-shou Hu, MD, PhD, Shou-jun Li, MD, PhD, Xin Wang, MD, PhD, Li-qing Wang, MD, PhD, Hui Xiong, MD, PhD, Li-huan Li, MD, Fu-xia Yan, MD, and $\mathrm{Xu}$ Wang, MD, PhD, Beijing, China

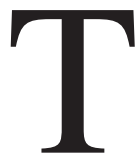

he Nikaidoh procedure, as an alternative to the Rastelli operation for dealing with transposition of the great arteries (TGA) with ventricular septal defect (VSD) and pulmonary stenosis (PS), could obtain a superior anatomic result. ${ }^{1,2}$ However, the extracardiac conduit is unable to grow and is inevitably calcified; thereafter, the patients required reoperation. We report our experience with a novel modification in which the native pulmonary valve was preserved to address these problems.

\section{Clinical Summary}

Since December 2004, 4 boys underwent surgical repair of TGA with VSD and PS at our heart center. The patients' demographic characteristics and clinical findings are presented in Table 1. All the patients had follow-up.

Definitive repair was carried out during moderate hypothermia with cardiopulmonary bypass. The ascending aorta and the pulmonary trunk were transected above the sinotublar conjunction. The coronary arteries were mobilized and detached. The aortic root and pulmonary root, including the semilunar valves, were excised beneath the annular level from the ventricles (Figure 1, A). In patients 2 and 4, who had atrioventricular discordance, the anterior part of the pulmonary annuli was untouched, and only the posterior part of the pulmonary root was dissected to avoid injury of the conduction tissue (Figure 1, $C)$. The subvalvular stenosis was relieved by resecting the conal septum. A Dacron patch and 5/0 Prolene running sutures (Ethicon, Inc, Somerville, NJ) were used to close the VSD. Subsequently, the detached aortic root was translocated posteriorly

From the Department of Cardiovascular Surgery, Cardiovascular Institute and Fu-Wai Hospital, Chinese Academy of Medical Sciences, Peking Union Medical College, Beijing, China.

Received for publication Feb 18, 2006; revisions received April 7, 2006; accepted for publication April 11, 2006.

Address for reprints: Sheng-shou $\mathrm{Hu}, \mathrm{MD}, \mathrm{PhD}$, Department of Cardiovascular Surgery, Cardiovascular Institute and Fu-Wai Hospital, Beijing 100037, P. R. China (E-mail: huss@163bj.com or xinwang_2002@hotmail.com).

J Thorac Cardiovasc Surg 2007;133:1090-2

$0022-5223 / \$ 32.00$

Copyright @ 2007 by The American Association for Thoracic Surgery doi:10.1016/j.jtcvs.2006.04.058 to the original pulmonary trunk position, and coronary arteries were reimplanted. After the Lecompte maneu-

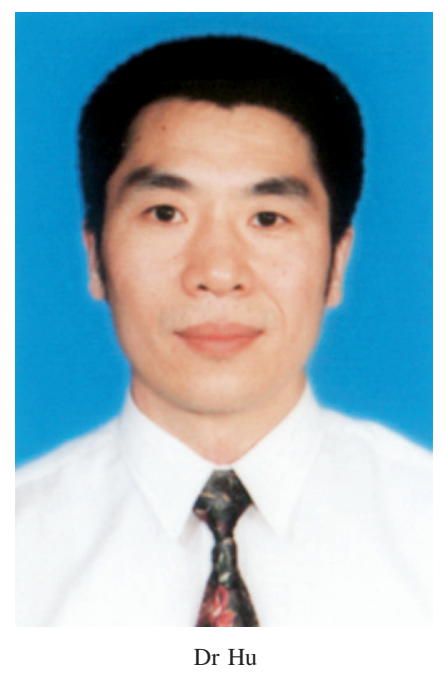
ver was performed, the aortic continuity was reestablished. The detached pulmonary root was then translocated anteriorly to the right ventricular outflow tract (RVOT). The pulmonary root was incised along its anterior wall, and a monocusp bovine jugular vein patch was used to enlarge the RVOT (Figure 1, $B$ ). Also, in the second and fourth patients, with atrioventricular discordance, an atrial switch operation (Senning procedure) was performed.

All patients survived the operation and were discharged from the hospital. There were no major complications in this series. The third patient was supported with extracorporeal membrane oxygenation because of a poor heart function on the third postoperative day; extracorporeal membrane oxygenation was weaned smoothly on the seventh postoperative day. All the patients had normal heart rhythm at discharge.

At a median follow-up of 5.5 months, all 4 patients were alive. Echocardiography demonstrated that all the patients had normal ventricular function. The median left ventricular ejection fraction value was $73 \%$ (range, 63\%-85\%). No residual aortic stenosis or insufficiency was found in any patient. Patients 2 and 4 had mild pulmonary insufficiency. Patient 4 had a $1.2-\mathrm{mm}$ residual VSD. All the patients had normal heart rhythm during the follow-up period.

\section{Discussion}

In 1984, the Nikaidoh procedure was introduced to correct TGA with VSD and PS. ${ }^{1}$ It involves aortic translocation and biventricular outflow tract reconstruction. The Nikaidoh procedure results in more normally aligned right and left ventricular outflow tracts, which theoretically should result in better intracardiac flow dynamics and should, in turn, result in improved outcomes over the longer term. However, growth incapability of the RVOT and pulmonary insufficiency are still main complications that require reoperation.

In comparison with the original technique of the Nikaidoh procedure, our method described herein has the following advantages. First, the pulmonary root with the preserved valve was dissected out and relocated to the normal anatomic position to the reconstructed RVOT, and the translocation of the native 
TABLE 1. The patients' demographic characteristics and clinical findings

\begin{tabular}{|c|c|c|c|c|c|c|}
\hline \multirow[b]{2}{*}{ Patient no. } & \multirow[b]{2}{*}{ Age (y) } & \multicolumn{2}{|c|}{ Pulmonary outflow obstruction } & \multirow[b]{2}{*}{ Morphologic diagnosis } & \multirow{2}{*}{$\begin{array}{l}\text { Previous performed } \\
\text { procedure }\end{array}$} & \multirow[b]{2}{*}{ Follow-up (mo) } \\
\hline & & Type & Gradient (mm Hg) & & & \\
\hline 1 & 2 & $\begin{array}{l}\text { Moderate subvalvular and } \\
\text { supervalvular; mild } \\
\text { valvular (bicuspid) }\end{array}$ & 76.1 & $\begin{array}{l}\text { TGA, perimembrane VSD, } \\
\text { PS, PDA }(1 \mathrm{AD}, \mathrm{R} ; 2 \mathrm{Cx})^{*}\end{array}$ & None & 13 \\
\hline 2 & 15 & Mild valvular (bicuspid) & 70 & $\begin{array}{l}\text { cTGA, perimembrane VSD, } \\
\text { PS }(1 \mathrm{R}, A D, C x)^{*}\end{array}$ & None & 8 \\
\hline 3 & 6 & $\begin{array}{l}\text { Severe subvalvular; mild } \\
\text { valvular }\end{array}$ & 60.8 & $\begin{array}{l}\text { TGA, subpulmonary VSD, } \\
\text { PS, BT shunt (occluded) } \\
(1 \mathrm{AD}, \mathrm{Cx} ; 2 \mathrm{R})^{*}\end{array}$ & Modified BT shunt & 3 \\
\hline 4 & 6 & $\begin{array}{l}\text { Subvalvular and moderate } \\
\text { valvular (bicuspid) }\end{array}$ & 74 & $\begin{array}{l}\text { Isolated dextrocardia, cTGA, } \\
\text { perimembrane VSD, PS, } \\
\text { PLSVC (1 AD, Cx; } 2 \mathrm{R})^{*}\end{array}$ & None & 1 \\
\hline
\end{tabular}

TGA, Transposition of the great arteries; VSD, ventricular septal defect; $P S$, pulmonary stenosis; $P D A$, patent ductus arteriosus; $A D$, anterior descending artery; $R$, right coronary artery; $C X$, circumflex artery; $c T G A$, corrected transposition of the great arteries; BT, Blalock-Taussig; PLSVC, persistent left superior vena cava. *Coronary Leiden convention.

pulmonary root would probably have growth potential and best preserve valve function. Thus this modification might minimize the pulmonary insufficiency and allow growth of the pulmonary root. However, this modification could be used only in those whose pulmonary roots have no more than moderate stenosis; otherwise, the severely hypoplastic pulmonary artery and annuli are unlikely to grow. Second, in our modified procedure, similar to that used by others, ${ }^{3}$ the coronary arteries are reimplanted. Two patients had difficult coronary artery anatomy (coronary patterns of patients 1 and 2, respectively; Table 1), and there were no adverse results related to the coronary insufficiency. Therefore we believe that by using this modified technique, aortic translocation could be performed in almost all the patients with difficult coronary patterns.

In summary, the modification of the Nikaidoh procedure that preserves the native pulmonary valve might minimize the postoperative pulmonary insufficiency. The procedure might also allow growth of the pulmonary root and therefore decrease the need for reoperation. However, the long-term results warrant further follow-up studies.

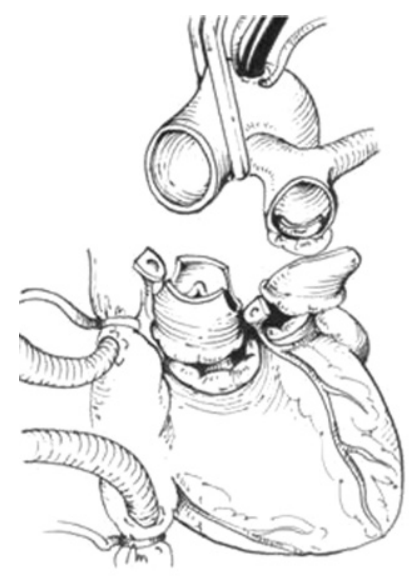

A

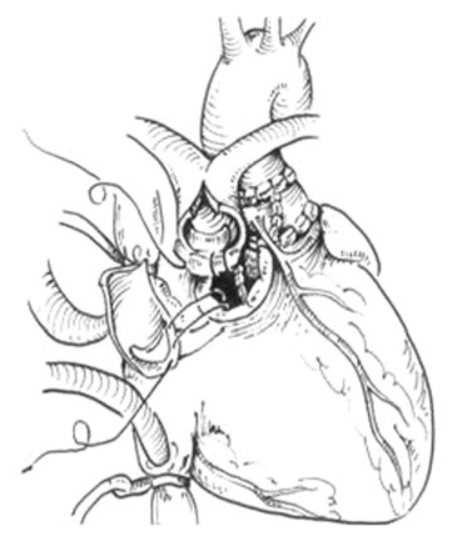

$\mathrm{B}$

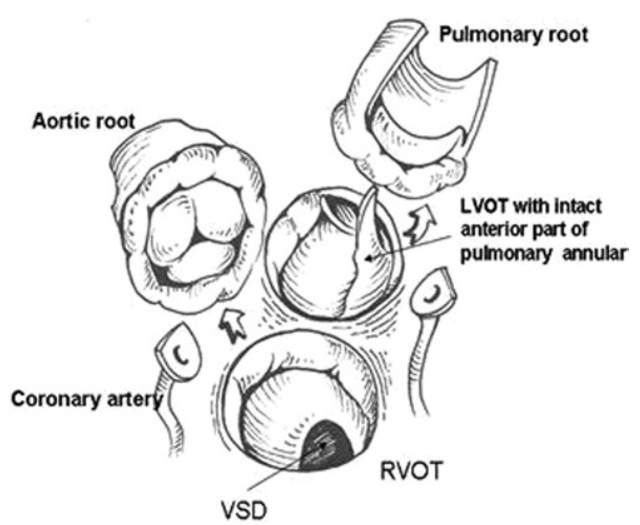

C

Figure 1. Diagram of the modification of the Nikaidoh procedure. A, The ascending aorta and the pulmonary trunk were transected. The coronary arteries were detached. The aortic and pulmonary roots were dissected out. B, The ventricular septal defect (VSD) was closed, and the aortic root was translocated to the left ventricular outflow tract (LVOT). The anteriorly translocated pulmonary root was enlarged. C, In a patient with atrioventricular discordance, the anterior part of the pulmonary annuli was untouched, and only the posterior part of the pulmonary root was dissected. RVOT, Right ventricular outflow tract. 


\section{References}

1. Nikaidoh H. Aortic translocation and biventricular outflow tract reconstruction. J Thorac Cardiovasc Surg. 1984;88:365-72.

2. Nikaidoh H, Leonard SR. Aortic translocation and biventricular outflow tract reconstruction for d-transposition associated with ventricular septal defect and pulmonary stenosis: a follow-up. Presented at: Third World Congress of Pediatric Cardiology and Cardiac Surgery; May 28, 2001; Toronto, Canada.

3. Morell OV, Jacobs PJ, Quintessenza AJ. Aortic translocation in the management of transposition of the great arteries with ventricular septal defect and pulmonary stenosis: Results and follow-up. Ann Thorac Surg. 2005;79:2089-93.

\section{Interruption of the aorta with multilobulated arch aneurysms: A new clinicopathologic entity}

Victor T. Tsang, FRCS, ${ }^{a}$ Philip J. Kilner, MD, ${ }^{\mathrm{b}}$ Tain-Yen Hsia, MD, ${ }^{a}$ Sian Hughes, MD, ${ }^{\mathrm{c}}$ and Magdi Yacoub, FRS, London and Middlesex, United Kingdom

$\Gamma$ he aortic arch occupies an extremely strategic position with a capacity to influence blood supply to virtually all parts of the body. In addition, the aortic arch is surrounded by many vital structures. To date, several congenital and acquired abnormalities affecting the aortic arch have been described and well characterized from the clinical and pathologic points of view. ${ }^{1}$

We here describe a "new" condition of complete or partial replacement of the aortic arch and some of its branches by multilobulated "aneurysms" that are interconnecting by a series of small openings and therefore are obstructive. In addition, we discuss its management and the possible developmental origin.

\section{Clinical Summaries}

Patient 1. A 26-year-old woman had a short history (approximately 6 months) of shortness of breath and diminished upper and lower limb pulses and a suprasternal pulsatile mass. Cardiovascular magnetic resonance (CMR) showed tapering of the aortic arch followed by interruption, bypassed by a $4 \times 5 \times 6-\mathrm{cm}$ multilobulated aneurysm located superior to the expected curve of the arch (Figure 1, $a$ and $b$ ). There was compression of the distal trachea. The tortuous distal aortic arch gave rise to anomalous, posteriorly located right and left subclavian arteries. No left common carotid artery was identified. The left brachiocephalic vein passed behind the ascending arch.

Repair was undertaken with deep hypothermic circulatory arrest at $15^{\circ} \mathrm{C}$. Postoperatively, the patient had transient right leg weakness, which resolved completely. Postoperative CMR study (Figure 1,c) demonstrated a patent graft and bilateral vertebral arterial flow to subclavian arteries with cerebral arterial steal. At 8 months' follow-up, she remained well, participating in sports with no signs of subclavian steal. Her pulses were strong and equal in all extremities.

Patient 2. A 47-year-old woman was known to have had a heart murmur since childhood but was well until 36 years of age, when she was evaluated for left upper extremity weakness, which was treated conservatively. Ten years later she began having chest pain and dyspnea on exertion. CMR showed coarctation of a right-sided aortic arch followed by a tortuous, multilobulated aneurysmal sac located to the right of the ex-

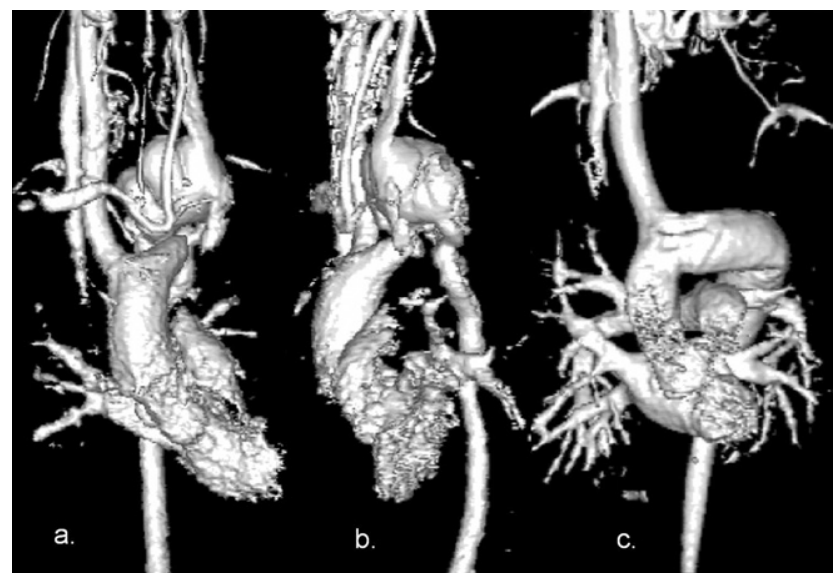

Figures 1a and b. Contrast-enhanced magnetic resonance angiography showing anteroposterior and left lateral views of the aneurysmal aortic arch of patient 1. c, Contrast-enhanced magnetic resonance angiography showing a left lateral view of the aneurysmal aortic arch of patient 1 after graft repair.

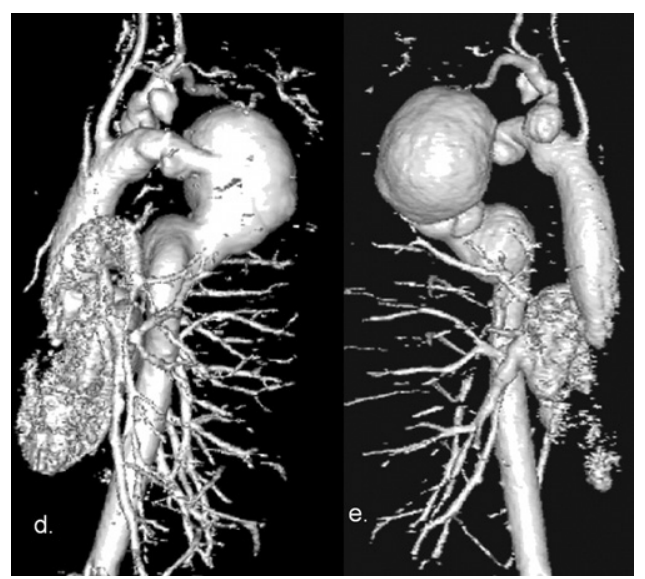

Figures 1d and e. Contrast-enhanced magnetic resonance angiography showing left lateral and right lateral views of the aneurysmal aortic arch of patient 2. 